\title{
Signal Integrity Analysis in Single and Bundled Carbon Nanotube Interconnects
}

\author{
Manoj Kumar Majumder, Nisarg D. Pandya, B. K. Kaushik, and S. K. Manhas \\ Microelectronics and VLSI Group, Department of Electronics and Communication Engineering, Indian Institute of Technology Roorkee, \\ Roorkee, Uttarakhand 247667, India \\ Correspondence should be addressed to Manoj Kumar Majumder; manojbesu@gmail.com
}

Received 29 March 2013; Accepted 11 July 2013

Academic Editor: Changxin Chen

Copyright (C) 2013 Manoj Kumar Majumder et al. This is an open access article distributed under the Creative Commons Attribution License, which permits unrestricted use, distribution, and reproduction in any medium, provided the original work is properly cited.

Carbon nanotube $(\mathrm{CNT})$ can be considered as an emerging interconnect material in current nanoscale regime. They are more promising than other interconnect materials such as $\mathrm{Al}$ or $\mathrm{Cu}$ because of their robustness to electromigration. This research paper aims to address the crosstalk-related issues (signal integrity) in interconnect lines. Different analytical models of single- (SWCNT), double- (DWCNT), and multiwalled CNTs (MWCNT) are studied to analyze the crosstalk delay at global interconnect lengths. A capacitively coupled three-line bus architecture employing CMOS driver is used for accurate estimation of crosstalk delay. Each line in bus architecture is represented with the equivalent RLC models of single and bundled SWCNT, DWCNT, and MWCNT interconnects. Crosstalk delay is observed at middle line (victim) when it switches in opposite direction with respect to the other two lines (aggressors). Using the data predicted by ITRS 2012, a comparative analysis on the basis of crosstalk delay is performed for bundled SWCNT/DWCNT and single MWCNT interconnects. It is observed that the overall crosstalk delay is improved by $40.92 \%$ and $21.37 \%$ for single MWCNT in comparison to bundled SWCNT and bundled DWCNT interconnects, respectively.

\section{Introduction}

Advancement of VLSI technology leads to the development of high-speed complex integrated circuits (ICs) in current nanoscale regime. Due to shrinking feature sizes and increasing clock frequency, interconnect plays an important role in determining the overall circuit performance. Therefore, in recent technology, interconnect delay dominates over the gate delay. The global interconnects are widely employed to distribute data, clock, power supply, and ground throughout the entire area of an IC. At global interconnect, most materials (such as $\mathrm{Al}$ or $\mathrm{Cu}$ ) are susceptible to electromigration due to higher current density. This electromigration problem substantially affects the reliability of high-speed VLSI circuits. To avoid such problems, researchers are forced to find an alternative solution for global VLSI interconnects.

Carbon nanotubes (CNTs) can be considered as alternative interconnect material in current nanoscale regime.
After discovery in 1991 [1], CNTs have received tremendous research interest for their unique mechanical [2], electrical [3], thermal [4], and chemical properties [5]. The $s p_{2}$ bonding in graphene is even stronger than the $s p_{3}$ bonds in diamond that gives CNTs extremely high mechanical strength [6]. The unique electrical and thermal properties are primarily due to the movement of electrons in one-dimensional (1D) systems. Due to the 1D movement, electrons can be scattered only in backward direction [5]. Therefore, mean free path $(m f p)$ in high-quality nanotubes is in the range of micrometer. This is in contrast to a three-dimensional (3D) metallic wire in which electrons can be backscattered by a series of small-angle scatterings, and therefore, $m f p s$ are in the range of few tens of nanometers [7]. Due to long $m f p$, CNTs can exhibit the ballistic transport phenomenon which is responsible for their outstanding electrical and thermal behaviour. Moreover, an isolated CNT can carry current densities up to $10^{9} \mathrm{~A} / \mathrm{cm}^{2}$ at an elevated temperature of 
$250^{\circ} \mathrm{C}$ [7], thereby eliminating electromigration reliability concern. Due to these extraordinary properties, CNTs are suitable for a variety of applications in the areas of microelectronics/nanoelectronics [8], spintronics [9], optics [10], material science [11], and mechanical [12] and biological fields [13].

CNTs, known as allotropes of carbon [1], are cylindrically rolled-up sheets of graphene. Graphene is a two-dimensional sheet of graphite that exhibits honeycomb lattice arrangement among the carbon atoms [6]. Based on the number of rolled-up graphene sheets, CNTs are classified into single(SWCNT) and multiwalled CNTs (MWCNT). Double-walled CNT (DWCNT) is a special type of MWCNT wherein only two concentrically rolled-up graphene sheets are present. Due to structural simplicity, SWCNTs have attracted more attention than DWCNTs and MWCNTs. Significant progress has been achieved in the characterization of interconnect performances of SWCNT bundle and MWCNT [7, 14]. For example, compact physical models were developed for MWCNTs and bundled SWCNTs for different number of conducting channels [15], and performance prediction of bundled SWCNTs for on-chip interconnects was demonstrated in [16]. Diameter-dependent model was analyzed for bundled SWCNT and MWCNT interconnects in [17], and their performances were compared [18] against $\mathrm{Cu} /$ low$\mathrm{k}$ wires for future high-performance ICs. Performance in terms of crosstalk is an important design concern in current nanoscale VLSI interconnects. Crosstalk in coupled lines can be broadly classified into two categories: (1) functional and (2) dynamic crosstalk [19, 20]. Under functional crosstalk category, victim line experiences a voltage spike when an aggressor line switches [21]. On the other hand, dynamic crosstalk is observed when aggressor and victim line switches simultaneously. A change in signal propagation delay is experienced under dynamic crosstalk when adjacent line (aggressor and victim) switches either in phase or out of phase. Using parallel SWCNTs or single MWCNT, crosstalkinduced delay and peak voltages were first observed by Rossi et al. [14] in three-line bus architecture. Again, in 2009, Chen et al. [22] reported that at high biasing voltages, SWCNT interconnect arrays were significantly affected by dynamic crosstalk delay.

Based on the interconnect geometry predicted by ITRS 2012 [23], propagation delay under the influence of dynamic crosstalk is analyzed for equivalent $R L C$ models of MWCNT and bundled SWCNT/DWCNT at global interconnect lengths. Using a capacitively coupled three-line bus architecture employed by metallic nanotube, it is observed that crosstalk delays are significantly improved in MWCNT as compared to the bundled SWCNT/DWCNT interconnects. The organization of this paper is as follows. Section 1 introduces the recent research scenario and briefs about the works carried. Based on the geometry and bundle arrangements, Section 2 describes the equivalent RLC models of bundled SWCNT/DWCNT and single MWCNT interconnects. A detailed description about the three-line bus architecture is provided in Section 3 whereas Section 4 analyzes the crosstalk delays for different single and bundled
CNT interconnects. Finally, Section 5 draws a brief summary of this paper.

\section{Geometry and Equivalent RLC Models}

This section presents the geometry and equivalent $R L C$ models of bundled SWCNT, bundled DWCNT, and single MWCNT interconnects. Figure 1(a) shows a bundled SWCNT (height $=h$ and width $=w$ ) that consists of numbers of SWCNTs with diameter $d$ and spacing $S_{p}$. The total number of SWCNTs in a bundle can be calculated as $[7,24]$

$$
\begin{array}{r}
n_{\mathrm{CNT}}=n_{W} n_{H}-\left(\frac{n_{H}}{2}\right), \quad n_{\mathrm{CNT}}=n_{W} n_{H}-\left(\frac{n_{H}-1}{2}\right) \\
\left(\text { if } n_{H}\right. \text { is an even and odd number, resp.), }
\end{array}
$$

where

$$
n_{W}=\left\lfloor\frac{w-d}{S_{p}}\right\rfloor+1, \quad n_{H}=\left\lfloor\frac{h-d}{S_{p}}\right\rfloor+1 .
$$

$n_{H}$ and $n_{W}$ are number of rows and columns, respectively, $n_{\mathrm{CNT}}$ is the total number of CNTs in bundle, and $\lfloor N\rfloor$ indicates the largest integer which is less than or equal to $N$. Similarly, Figure 1(b) exhibits the arrangement of bundled DWCNT that considers $n$ number of DWCNTs and are calculated using (1) and (2). DWCNT can be considered as the simplest geometry of an MWCNT. An MWCNT consists of $n$ number of shells with different diameters of $D_{1}, D_{2}, D_{3}, \ldots, D_{n}$, where $D_{1}$ and $D_{n}$ are the inner and outer shell diameters, respectively, as shown in Figure 1(c). In current fabrication process, the intershell spacing of MWCNT can be calculated as [14]

$$
S_{i}=\frac{D_{n}-D_{n-1}}{2} \approx 0.34 \mathrm{~nm} .
$$

Based on geometry and bundle arrangements, the equivalent RLC models of bundled SWCNT, bundled DWCNT and single MWCNT are shown in Figures 2, 3, and 4, respectively. The total bundle resistance can be divided into two categories: $R_{l-b}$ and $R_{d-b}$, depending on and independent of the bundle lengths, respectively. Thus, $R_{l-b}$ and $R_{d-b}$ can be expressed as [7]

$$
R_{l-b}=\frac{\left(R_{C}+R_{\mathrm{Q}}\right)}{n_{\mathrm{CNT}}}, \quad R_{d-b}=\frac{R_{\mathrm{Q}}}{n_{\mathrm{CNT}}},
$$

where $R_{C}$ is the metal nanotube contact resistance with a typical value of $3.2 \mathrm{~K} \Omega$ and $R_{\mathrm{Q}}$ is the fundamental quantum resistance that can be expressed as [25]

$$
R_{\mathrm{Q}}=\frac{h}{4 e^{2}} \approx 6.45 \mathrm{~K} \Omega \text {. }
$$

Additionally, the bundled DWCNT and single MWCNT consider intershell tunneling conductance $\left(G_{t}\right)$ which is primarily due to the electron tunnel transport phenomenon between two shells [26]. Apart from this, the total bundle 


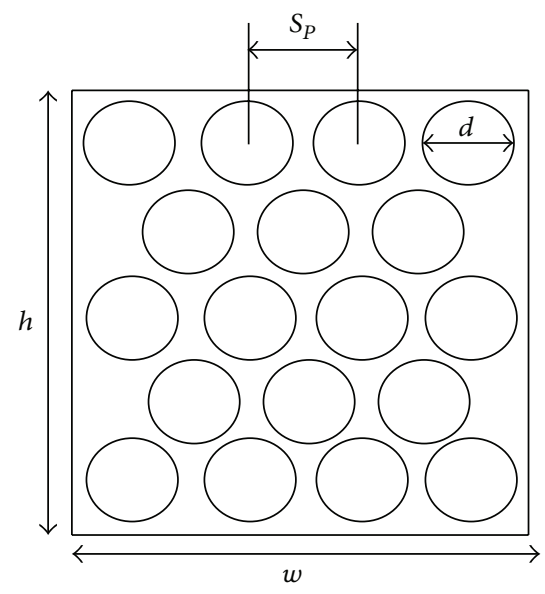

(a)

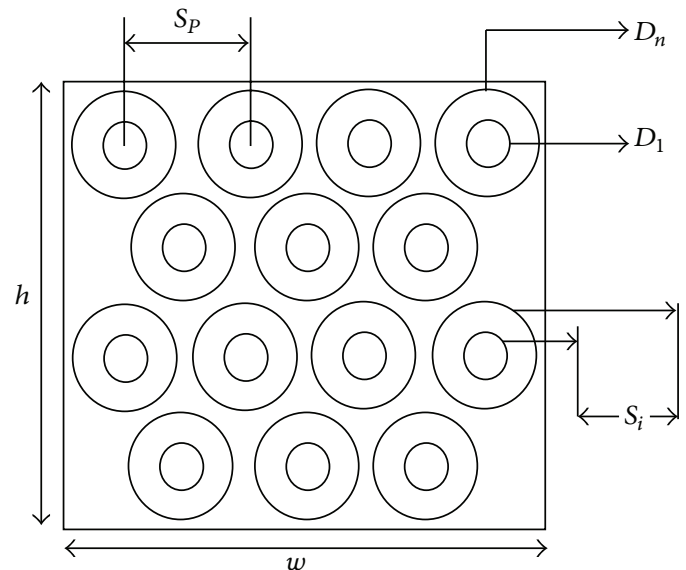

(b)

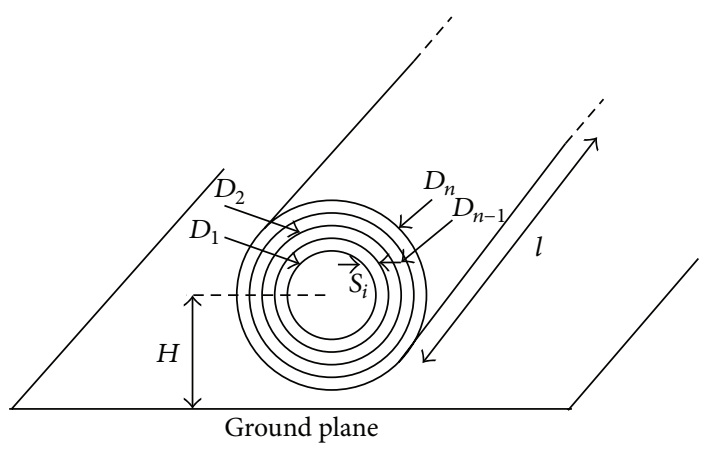

(c)

Figure 1: Geometry of (a) bundled SWCNT, (b) bundled DWCNT, and (c) single MWCNT.

$R_{l-b} / 2 \quad R_{d-b} / 2 \quad L_{K} / 8 n_{\mathrm{CNT}} L_{K} / 8 n_{\mathrm{CNT}} \quad R_{d-b} / 2 \quad R_{l-b} / 2$

'WWWWW

$$
C_{\mathrm{ES}-b} \frac{I}{T_{T}}
$$

FIGURE 2: Equivalent RLC model of bundled SWCNT interconnects.

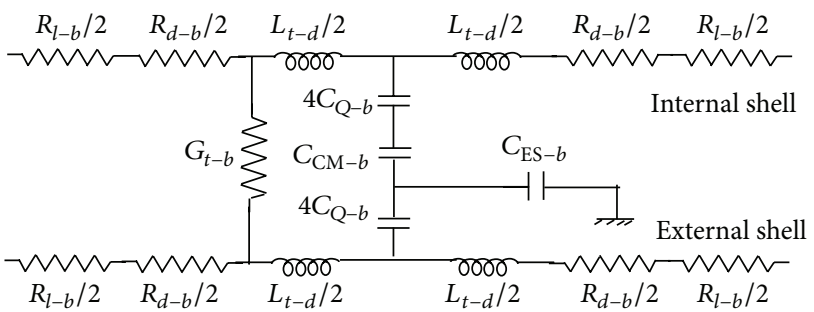

FIGURE 3: Equivalent RLC model of bundled DWCNT interconnects.

inductance $\left(L_{t-d}\right)$ is the summation of mutual and kinetic inductance of CNTs. Magnetic or mutual inductance is measured from magnetic field of an isolated current carrying wire having some distance " $h$ " from the ground plane, whereas kinetic inductance is mainly due to the charge carrier inertia $[25,27]$. Due to the unique band structure [5], kinetic inductance of CNTs dominates over the mutual inductance. Therefore, the equivalent model of Figure 4 consists of only kinetic inductance that appears as $L_{K} / 4$ due to four conducting channels in CNTs. The total bundle inductance $\left(L_{t-d}\right)$ can be formulated as [25]

$$
L_{t-d}=\frac{\left(L_{M}+L_{K}\right)}{n_{\mathrm{CNT}}} ; \quad \text { where } L_{K}=\frac{h}{2 e^{2} v_{F}},
$$

where $v_{F} \approx 8 \times 10^{5} \mathrm{~m} / \mathrm{s}$ is the Fermi velocity of CNT and graphene. The equivalent capacitance in CNT can be divided into (1) electrostatic capacitance $\left(C_{\mathrm{ES}}\right)$ that appears between the CNT and ground plane and (2) quantum capacitance $\left(C_{Q}\right)$ that exists due to the finite density of states of electrons. Additionally, a coupling capacitance $\left(C_{\mathrm{CM}}\right)$ occurs between each shell in bundled DWCNT and single MWCNT interconnects (Figures 3 and 4). $C_{\mathrm{CM}}$ primarily depends on the diameter of adjacent shells and can be expressed as [14]

$$
C_{\mathrm{CM}}=\frac{2 \pi \varepsilon}{\ln \left(D_{n} / D_{n-1}\right)}, \quad C_{\mathrm{CM}-b}=C_{\mathrm{CM}} \cdot n_{\mathrm{CNT}} .
$$




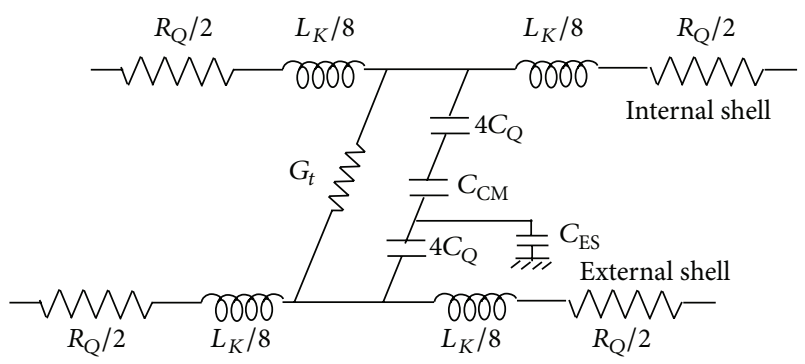

FIGURE 4: Equivalent RLC model of single MWCNT interconnects.

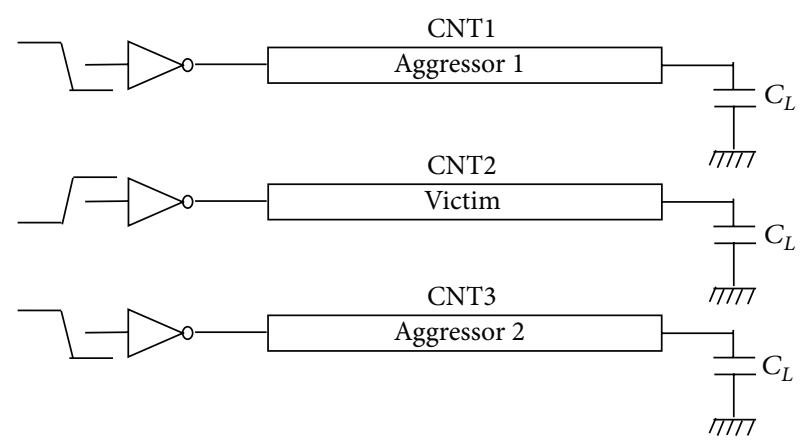

FIGURE 5: Three-line bus architecture made of three parallel CNTs (bundled SWCNTs/bundled DWCNTs/single MWCNTs).

\section{Three-Line Bus Architecture}

Propagation delay under the influence of dynamic crosstalk is analyzed for bundled SWCNT/DWCNT and single MWCNT interconnects using capacitively coupled three-line bus architecture [14] as shown in Figure 5. Out of these three lines, the middle one is referred to as victim while the other two as aggressors. Each interconnect line in bus architecture is represented using the equivalent $R L C$ models of bundled SWCNT (Figure 2), bundled DWCNT (Figure 3), and single MWCNT (Figure 4). A CMOS driver with supply voltage $1 \mathrm{~V}$ is used to drive the interconnect line. The primary characteristics of CMOS driver is that it operates partially in linear region and partially in saturation region during switching. But a transistor can be modeled by a resistor only in the linear region. In saturation region, the transistor is more accurately modeled as a current source with parallel high resistance $[19,28]$.

HSPICE simulations are performed for similar transition at aggressor lines when victim line experiences an opposite signal transition. Using the model parameters suggested by ITRS 2012 [23], crosstalk delay is performed for different interconnect lengths ranging from $800 \mu \mathrm{m}$ to $2000 \mu \mathrm{m}$.

\section{Signal Integrity in Bus Architecture}

This section analyzes the crosstalk delay (signal integrity) for bundled SWCNT/DWCNT and single MWCNT using capacitively coupled three-line bus architecture. For global interconnects, such as clock line, interconnect lengths can

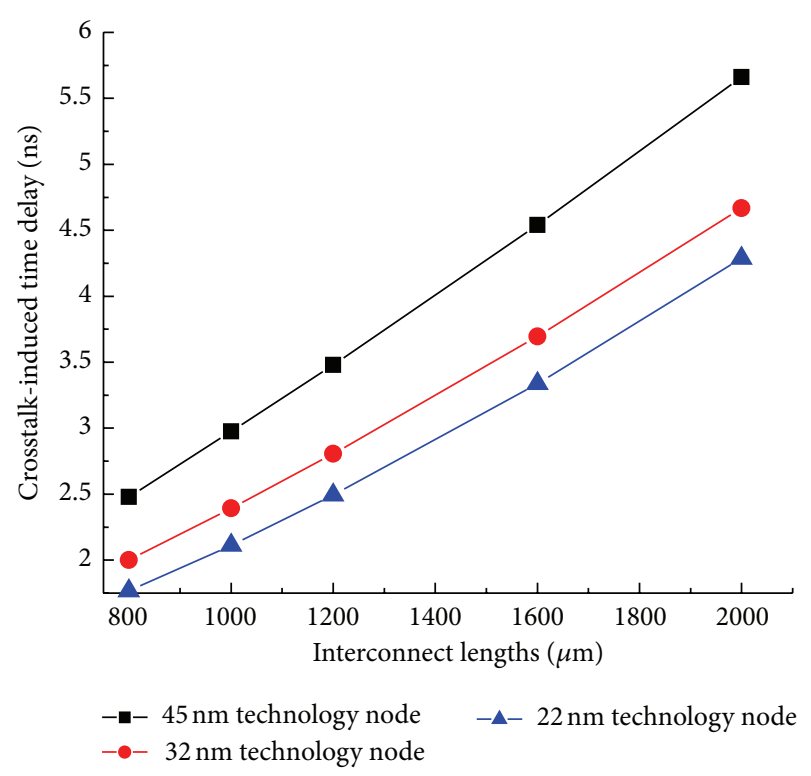

FIGURE 6: Crosstalk delay at different technology nodes for bundled SWCNT interconnects.

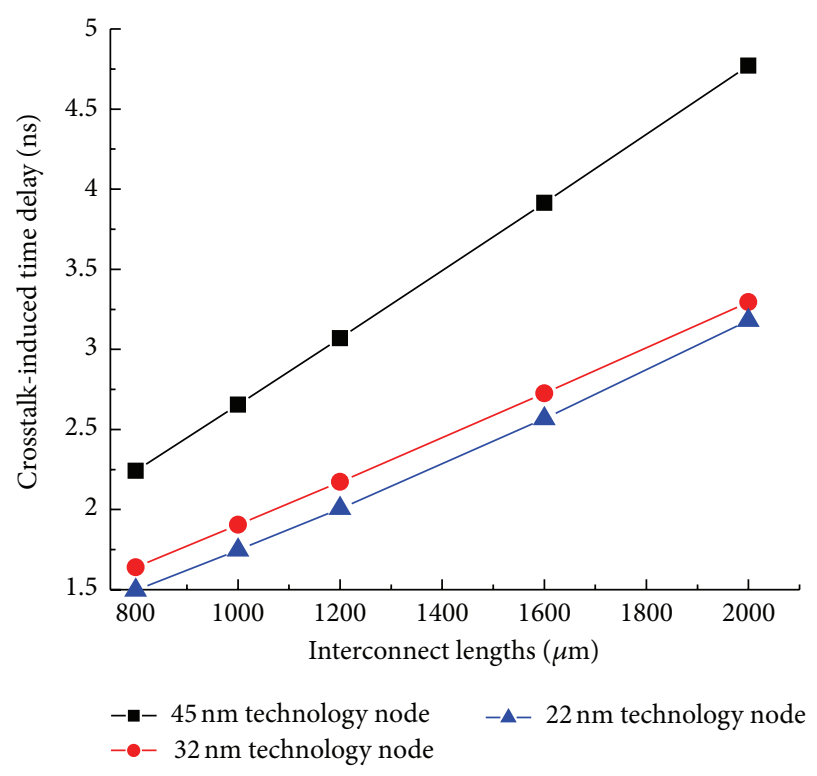

FIGURE 7: Crosstalk delay at different technology nodes for bundled DWCNT interconnects.

reach up to several millimeters. Using $22 \mathrm{~nm}, 32 \mathrm{~nm}$, and $45 \mathrm{~nm}$ technology nodes, Figures 6, 7, and 8 demonstrate the crosstalk delay for bundled SWCNT, bundled DWCNT, and single MWCNT interconnects, respectively. It is observed that the crosstalk delays of different single and bundled CNTs are considerably reduced for lower technology nodes. The primary reason is the reduced capacitive effect that has major impact on the dynamic crosstalk delay. As technology scales down, bundle width and height are reduced 
TABLE 1: Crosstalk delays for different CNT structures at $45 \mathrm{~nm}$ technology node.

\begin{tabular}{lccccc}
\hline \multirow{2}{*}{ Interconnect lengths $(\mu \mathrm{m})$} & \multicolumn{3}{c}{ Crosstalk delay $(\mathrm{ns})$ for } & \multicolumn{3}{c}{$\begin{array}{c}\text { Percentage improvement for single } \\
\text { MWCNT with respect to }\end{array}$} \\
& Bundled SWCNT & Bundled DWCNT & Single MWCNT & Bundled SWCNT & Bundled DWCNT \\
\hline 800 & 2.4793 & 1.9411 & 1.5541 & 37.32 & 19.94 \\
1000 & 2.9758 & 2.4539 & 1.8295 & 38.53 & 3.45 \\
1200 & 3.4801 & 3.0701 & 2.1088 & 39.41 & 31.31 \\
1600 & 4.5406 & 4.1137 & 2.6315 & 42.04 & 36.03 \\
2000 & 5.6611 & 4.9714 & 3.0421 & 46.26 & 38.81 \\
\hline
\end{tabular}

TABLE 2: Crosstalk delays for different CNT structures at $32 \mathrm{~nm}$ technology node.

\begin{tabular}{lccccc}
\hline \multirow{2}{*}{ Interconnect lengths $(\mu \mathrm{m})$} & \multicolumn{3}{c}{ Crosstalk delay (ns) for } & \multicolumn{2}{c}{$\begin{array}{c}\text { Percentage improvement for single } \\
\text { MWCNT with respect to }\end{array}$} \\
& Bundled SWCNT & Bundled DWCNT & Single MWCNT & Bundled SWCNT & Bundled DWCNT \\
\hline 800 & 2.0011 & 1.6396 & 1.3777 & 31.15 & 15.97 \\
1000 & 2.3925 & 1.9046 & 1.5977 & 33.22 & 22 \\
1200 & 2.8063 & 2.1725 & 1.6818 & 40.07 & 23.11 \\
1600 & 3.6958 & 2.7259 & 2.0910 & 43.42 & 23.29 \\
2000 & 4.6674 & 3.2954 & 2.3423 & 49.81 & 28.92 \\
\hline
\end{tabular}

TABLE 3: Crosstalk delays for different CNT structures at $22 \mathrm{~nm}$ technology node.

\begin{tabular}{|c|c|c|c|c|c|}
\hline \multirow[t]{2}{*}{ Interconnect lengths $(\mu \mathrm{m})$} & \multicolumn{3}{|c|}{ Crosstalk delay (ns) for } & \multicolumn{2}{|c|}{$\begin{array}{l}\text { Percentage improvement for single } \\
\text { MWCNT with respect to }\end{array}$} \\
\hline & Bundled SWCNT & Bundled DWCNT & Single MWCNT & Bundled SWCNT & Bundled DWCNT \\
\hline 800 & 1.7654 & 1.4955 & 1.3225 & 25.08 & 11.56 \\
\hline 1000 & 2.1123 & 1.7474 & 1.5203 & 28.02 & 12.99 \\
\hline 1200 & 2.4924 & 2.0064 & 1.6252 & 34.79 & 18.99 \\
\hline 1600 & 3.3366 & 2.5674 & 2.0198 & 39.46 & 21.32 \\
\hline 2000 & 4.2875 & 3.1796 & 2.2960 & 46.44 & 28.63 \\
\hline
\end{tabular}

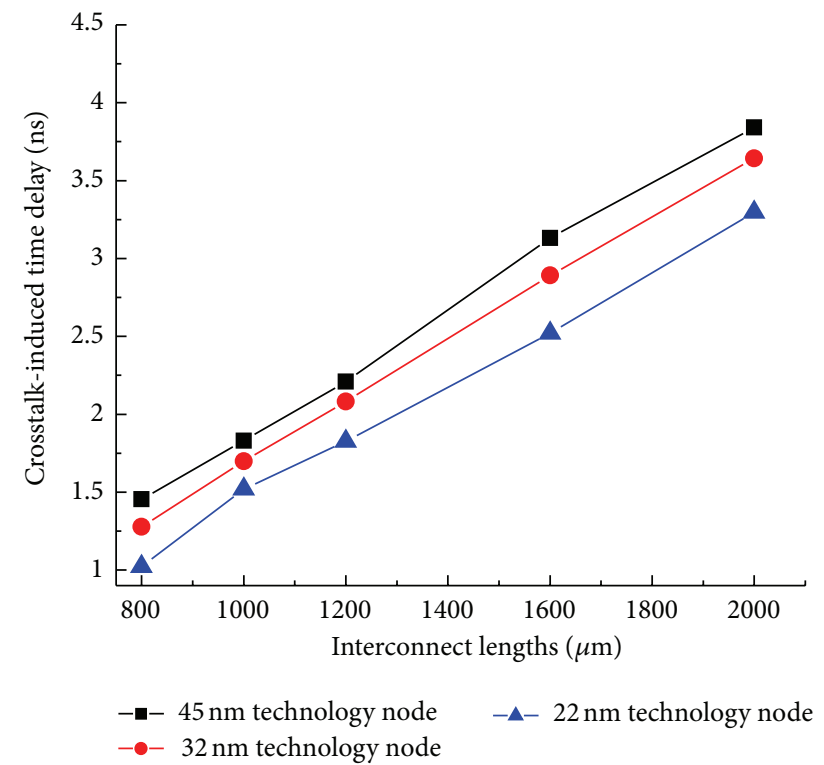

FIGURE 8: Crosstalk delay at different technology nodes for single MWCNT interconnects. which results in lesser number of CNTs and shells in bundled SWCNT/DWCNT and single MWCNT, respectively. The value of capacitive parasitic primarily depends on the number of CNTs in bundle and shells in MWCNT. Thus, the crosstalk delay is effectively reduced for lesser number of SWCNTs/DWCNTs in bundle and shells in MWCNTs at global interconnect lengths as indicated in Figures 6, 7, and 8 .

Tables 1, 2, and 3 summarize the crosstalk delay for different single and bundled CNTs at $45 \mathrm{~nm}, 32 \mathrm{~nm}$, and $22 \mathrm{~nm}$ technology nodes, respectively. It is observed that the overall crosstalk delay is improved by $40.97 \%$ and $21.37 \%$ in single MWCNT as compared to bundled SWCNT and bundled DWCNT interconnects, respectively. Irrespective of technology nodes, crosstalk delay is significantly reduced for MWCNT at higher interconnect lengths. The reason behind this reduction is the lower value of capacitive parasitic, that effectively reduces for single MWCNT in comparison to bundled SWCNT/DWCNT interconnects at specified technology node. Therefore, it can be concluded that a single MWCNT is more promising than bundled SWCNT/DWCNT in current nanoscale technology. 


\section{Conclusion}

This research paper analyzed the crosstalk delay for bundled SWCNT/DWCNT and single MWCNT interconnects by using coupled three-line bus architecture. The RLC models of single and bundled CNTs have been extended to address the geometry of the bus architecture and the CMOS driver is used to drive the interconnect lines. Dynamic crosstalk delay has been analyzed for worst-case scenario when the victim line is switched in opposite direction with respect to the aggressors. It has been observed that MWCNT exhibits a lower parasitic capacitance as compared to bundled SWCNT/DWCNT. Thus, the overall crosstalk delay is reduced by $40.92 \%$ and $21.37 \%$ for single MWCNT interconnects as compared to the bundled SWCNT and bundled DWCNT, respectively. Therefore, MWCNTs can be predicted as one of the most promising materials for the future global VLSI interconnects.

\section{References}

[1] R. Satio, G. Dresselhaus, and S. Desselhaus, Physical Properties of Carbon Nanotubes, Imperial College Press, London, UK, 1998.

[2] S. Berber, Y.-K. Kwon, and D. Tománek, "Unusually high thermal conductivity of carbon nanotubes," Physical Review Letters, vol. 84, no. 20, pp. 4613-4616, 2000.

[3] B. Q. Wei, R. Vajtai, and P. M. Ajayan, "Reliability and current carrying capacity of carbon nanotubes," Applied Physics Letters, vol. 79, no. 8, pp. 1172-1174, 2001.

[4] P. G. Collins, M. Hersam, M. Arnold, R. Martel, and P. Avouris, "Current saturation and electrical breakdown in multiwalled carbon nanotubes," Physical Review Letters, vol. 86, no. 14, pp. 3128-3131, 2001.

[5] A. Javey and J. Kong, Carbon Nanotube Electronics, Springer, New York, NY, USA, 2009.

[6] H. Li, C. Xu, N. Srivastava, and K. Banerjee, "Carbon nanomaterials for next-generation interconnects and passives: physics, status, and prospects," IEEE Transactions on Electron Devices, vol. 56, no. 9, pp. 1799-1821, 2009.

[7] S.-N. Pu, W.-Y. Yin, J.-F. Mao, and Q. H. Liu, "Crosstalk prediction of single- and double-walled carbon-nanotube (SWCNT/ DWCNT) bundle interconnects," IEEE Transactions on Electron Devices, vol. 56, no. 4, pp. 560-568, 2009.

[8] P. Avorious, Z. Chen, and V. Perebeions, "Carbon-based electronics," Nature Nanotechnology, vol. 2, pp. 605-613, 2007.

[9] K. Tsukagoshi, B. W. Alphenaar, and H. Ago, "Coherent transport of electron spin in a ferromagnetically contacted carbon nanotube," Nature, vol. 401, no. 6753, pp. 572-574, 1999.

[10] J. A. Misewich, R. Martel, P. Avouris, J. C. Tsang, S. Heinze, and J. Tersoff, "Electrically induced optical emission from a carbon nanotube FET,' Science, vol. 300, no. 5620, pp. 783-786, 2003.

[11] N. Wang, Z. K. Tang, G. D. Li, and J. S. Chen, "Materials science: single-walled $4 \AA$ carbon nanotube arrays," Nature, vol. 408, no. 6808, pp. 50-51, 2000.

[12] M.-F. Yu, O. Lourie, M. J. Dyer, K. Moloni, T. F. Kelly, and R. S. Ruoff, "Strength and breaking mechanism of multiwalled carbon nanotubes under tensile load," Science, vol. 287, no. 5453, pp. 637-640, 2000.

[13] F. Lu, L. Gu, M. J. Meziani et al., "Advances in bioapplications of carbon nanotubes," Advanced Materials, vol. 21, no. 2, pp. 139152, 2009.
[14] D. Rossi, J. M. Cazeaux, C. Metra, and F. Lombardi, "Modeling crosstalk effects in CNT bus architectures," IEEE Transactions on Nanotechnology, vol. 6, no. 2, pp. 133-145, 2007.

[15] A. Naeemi and J. D. Meindl, "Compact physical models for multiwall carbon-nanotube interconnects," IEEE Electron Device Letters, vol. 27, no. 5, pp. 338-340, 2006.

[16] A. Nieuwoudt and Y. Massoud, "Evaluating the impact of resistance in carbon nanotube bundles for VLSI interconnect using diameter-dependent modeling techniques," IEEE Transactions on Electron Devices, vol. 53, no. 10, pp. 2460-2466, 2006.

[17] S. Haruehanroengra and W. Wang, "Analyzing conductance of mixed carbon-nanotube bundles for interconnect applications," IEEE Electron Device Letters, vol. 28, no. 8, pp. 756-759, 2007.

[18] K.-H. Koo, H. Cho, P. Kapur, and K. C. Saraswat, "Performance comparisons between carbon nanotubes, optical, and $\mathrm{Cu}$ for future high-performance on-chip interconnect applications," IEEE Transactions on Electron Devices, vol. 54, no. 12, pp. 32063215, 2007.

[19] B. K. Kaushik, S. Sarkar, R. P. Agarwal, and R. C. Joshi, "An analytical approach to dynamic crosstalk in coupled interconnects," Microelectronics Journal, vol. 41, no. 2-3, pp. 85-92, 2010.

[20] M. K. Majumder, N. D. Pandya, B. K. Kaushik, and S. K. Manhas, "Dynamic crosstalk effect in mixed CNT bundle interconnects," IET Electronics Letters, vol. 48, pp. 384-385, 2012.

[21] J. M. Rabaey, Digital Integrated Circuits, A Design Perspective, Prentice Hall, Englewood Cliffs, NJ, USA, 1996.

[22] W. C. Chen, W.-Y. Yin, L. Jia, and Q. H. Liu, "Electrothermal characterization of single-walled carbon nanotube (SWCNT) interconnect arrays,' IEEE Transactions on Nanotechnology, vol. 8, no. 6, pp. 718-728, 2009.

[23] “2012 International Technology Roadmap for Semiconductors," http://public.itrs.net/.

[24] M. K. Majumder, N. D. Pandya, B. K. Kaushik, and S. K. Manhas, "Analysis of MWCNT and bundled SWCNT interconnects: impact on crosstalk and area," Electron Device Letters, vol. 33, pp. 1180-1182, 2012.

[25] P. J. Burke, "Luttinger liquid theory as a model of the gigahertz electrical properties of carbon nanotubes," IEEE Transactions on Nanotechnology, vol. 1, pp. 129-144, 2002.

[26] B. Bourlon, C. Miko, L. Forró, D. C. Glattli, and A. Bachtold, "Determination of the intershell conductance in multiwalled carbon nanotubes," Physical Review Letters, vol. 93, no. 17, Article ID 176806, 4 pages, 2004.

[27] A. Naeemi and J. D. Meindl, "Design and performance modeling for single-walled carbon nanotubes as local, semiglobal, and global interconnects in gigascale integrated systems," IEEE Transactions on Electron Devices, vol. 54, no. 1, pp. 26-37, 2007.

[28] M. K. Majumder, N. D. Pandya, B. K. Kaushik, and S. K. Manhas, "Analysis of crosstalk delay and area for MWNT and bundled SWNT for global VLSI Interconnects," in Proceedings of the 13th IEEE International Symposium on Quality Electronic Design (ISQED '12), pp. 291-297, Santa Clara, Calif, USA, 2012. 

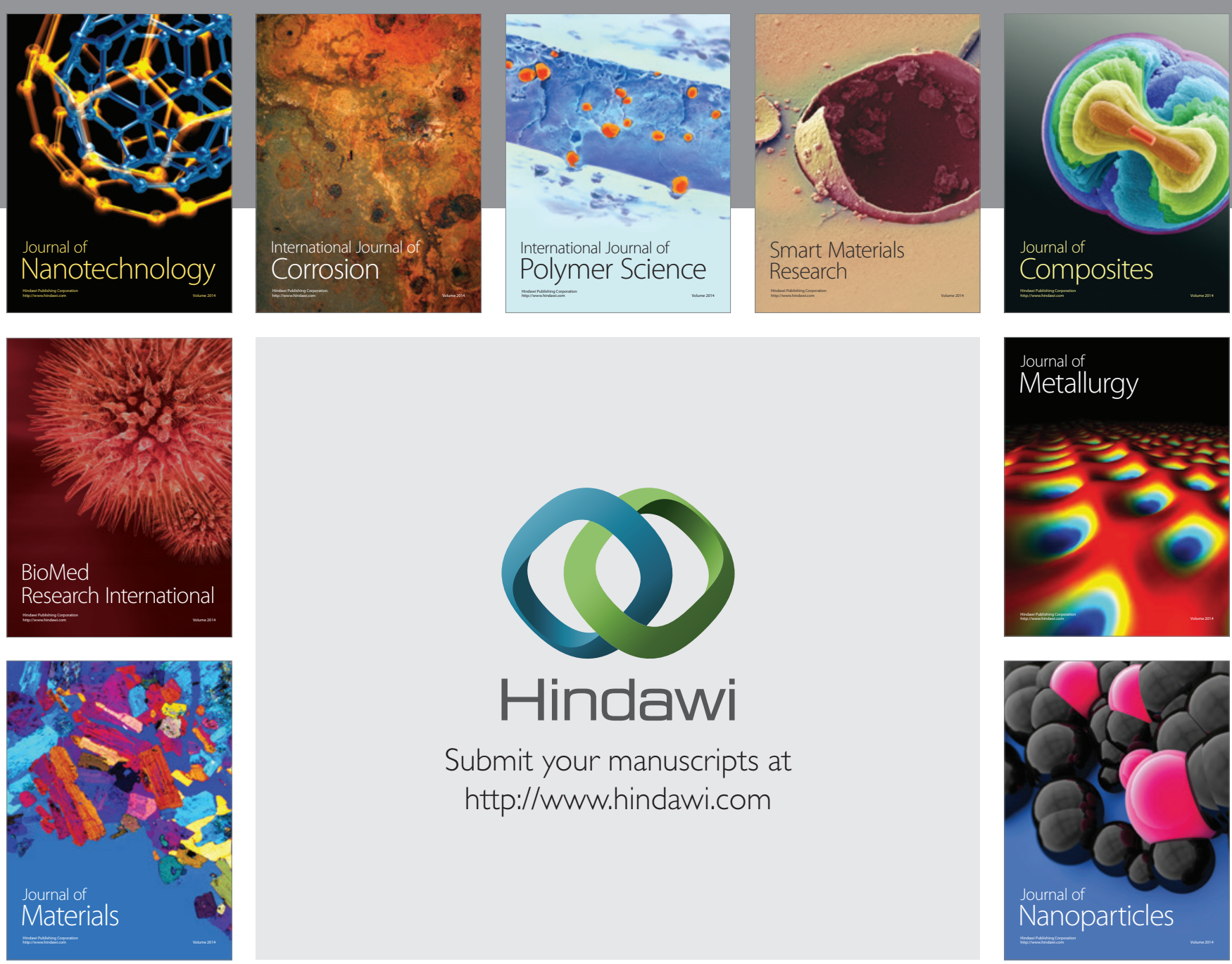

Submit your manuscripts at http://www.hindawi.com
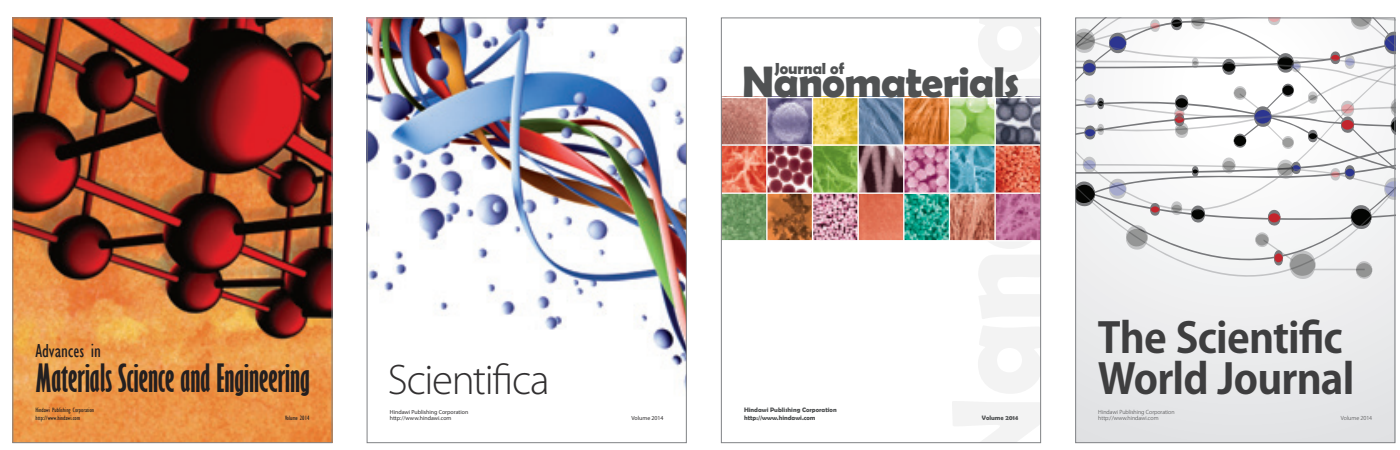

\section{The Scientific World Journal}
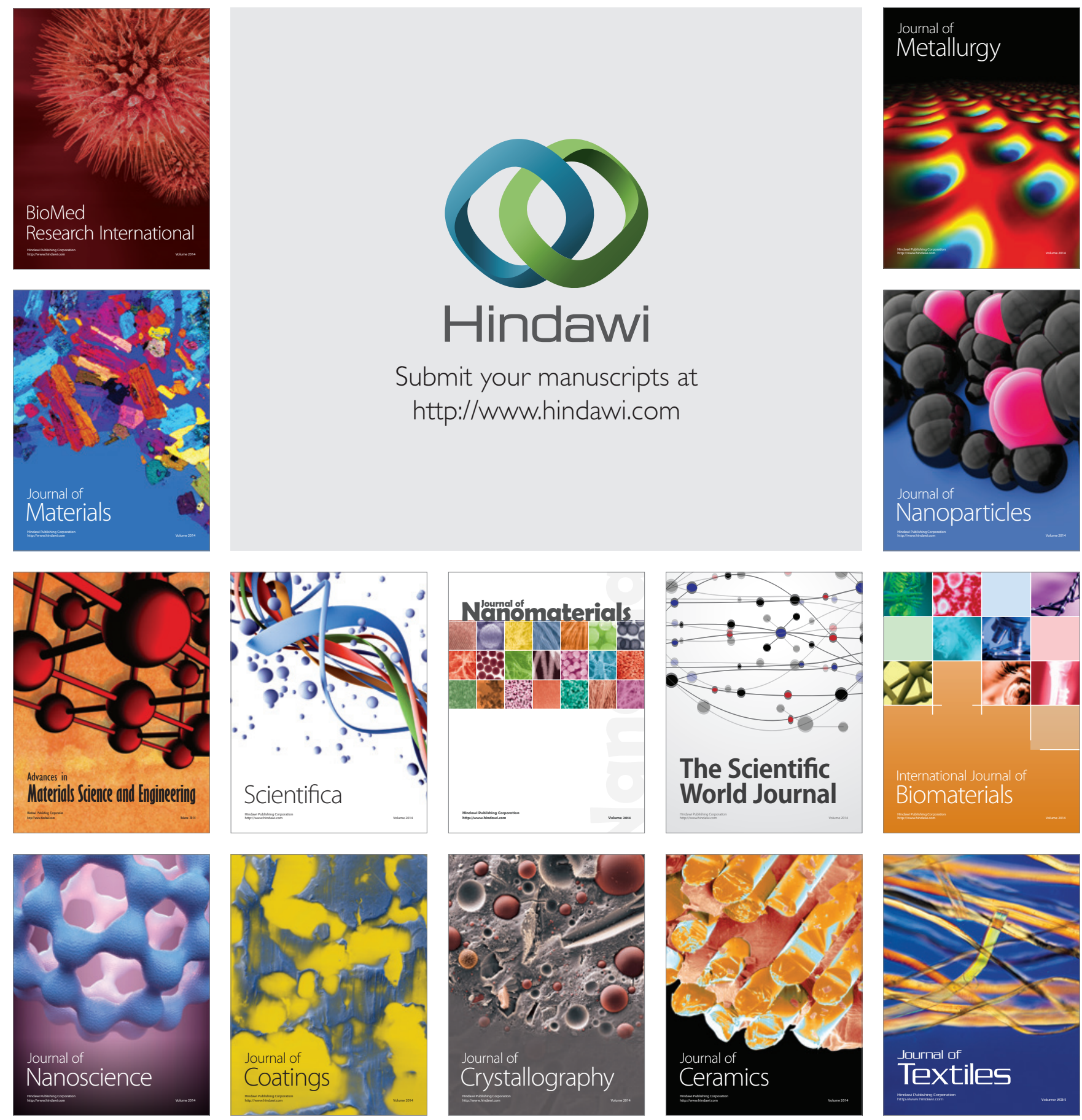